\title{
Large-eddy simulation of aircraft wake vortex deformation and topology
}

\author{
I Hennemann* and F Holzäpfel \\ Deutsches Zentrum für Luft- und Raumfahrt, Institut für Physik der Atmosphäre, Oberpfaffenhofen, Germany
}

The manuscript was received on 3 August 2010 and was accepted after revision for publication on 9 February 2011.

DOI: $10.1177 / 0954410011402257$

\begin{abstract}
On the one hand, it is known from visual observations, Lidar measurements, and numerical simulations that aircraft wake vortices may live significantly longer than anticipated by todays standard regulations of air traffic control. On the other hand, the initially counterrotating, parallel vortex pair deforms quickly, which reduces the impact time of adverse forces and moments on encountering aircraft. Therefore, large-eddy simulations of wake vortex evolution in different meteorological conditions are conducted in order to analyse the physics of vortex deformation. A new post-processing algorithm has been developed that at first determines the three-dimensional path of the vortex core line and then computes piecewise curvature radii as a measure of vortex deformation. It is found that larger turbulence levels and neutral to weakly stable temperature stratification particularly support vortex ring formation. The vortex ring regime is characterized by a reduced descent rate and by a surprising variability of its core radius. It is shown that the varying core size directly affects the evolution of radii-averaged circulation. Comparisons with field experiments indicate good agreement with the considerable life time of the vortex rings and topology.
\end{abstract}

\section{INTRODUCTION}

Aircraft wake vortices represent a potential danger for following aircraft, especially in the vicinity of airports where the separation distance between two consecutive aircraft is relatively small. It has often been observed that wake vortices can live significantly longer than anticipated by ICAO wake vortex separations (e.g. references $[\mathbf{1}, \mathbf{2}])$. The reason for the underestimation of vortex lifetime in many numerical studies and Lidar measurements is related to vortex distortion and the fact that because of simplicity and because it is most relevant for operational use, these methods only detect the fraction of the vortices oriented in flight direction. A clue for the existence of long-lived wake vortices above runways is given by the observation that aircraft frequently experience

*Corresponding author: Deutsches Zentrum für Luft- und Raumfahrt, Institut für Physik der Atmosphäre,

Oberpfaffenhofen 82234, Germany.

email: ingo.hennemann@dlr.de unexpected rolling moments shortly before landing [3].

The increasing demand on airport capacity, however, requires optimized landing intervals. It is commonly believed that todays regulations are too conservative for many meteorological situations [4], but the distance between two aircraft can only be decreased if any loss of safety is excluded. Although at European airports it appears possible again to build new runways, pressure on airlines and pilots will persist to reduce separation distances as much as possible due to economical reasons, in particular when delays have to be made up.

The influence of wake vortices on following aircraft is known since the 1950s, but became relevant with the first take-off of the Boeing B747 in 1969. In 1970, the theoretical basis for vortex deformation was set by Crow [5] who used a linear stability approach to analyse the temporal evolution of a counter-rotating, fully rolled-up vortex pair. He found a cooperative instability with sinusoidal oscillations where the dominant mode is symmetric and grows exponentially in 
planes inclined by $45^{\circ}$ to the horizontal. This analysis was followed by many theoretical, experimental and later numerical investigations, which provided a more detailed understanding of wake vortex properties and dynamics (e.g. references $[4,6,7])$. In order to exploit research results operationally, the collected knowledge was used to design an engineering vortex model which predicts the temporal evolution of circulation and position [8]. Refined wake-vortex realtime models have been suggested subsequently [9-12].

So far, circulation from large-eddy simulations (LES) has been determined only from the vorticity component directed in flight direction whereas vorticity in spanwise or vertical direction has been ignored. In water tank experiments [1], side-view observations of the vortex pair (detecting circulation perpendicular to spanwise direction) yielded a significant amount of circulation at late instants of time, which implies the formation of stable vortex rings [2]. References [13] and [14] describe the vortex reconnection process by analysing direct numerical simulations (DNS), but the subsequent development of the wake vortex ring has not been considered so far.

This paper investigates the evolution of a symmetric counter-rotating vortex pair in terms of circulation, descent, and curvature by evaluating LES. For this purpose a post-processing algorithm has been developed, which identifies the vortex core line independently of its orientation. A second algorithm derives curvature radii from the identified segments of the vortex core line in order to quantitatively characterize the vortex topology. The impact of the geometrical vortex shape on the descent behaviour of the vortices is considered. Furthermore, the evolution of the vortex properties core radius, tangential velocity, and axial velocity are analysed which affect vortex evolution in its ring regime. The effects of meteorological conditions like stable temperature stratification and surrounding atmospheric turbulence are significant and consequently considered explicitly by conducting pre-runs in order to generate background temperature and velocity fields which represent the atmospheric environment realistically.

\section{NUMERICAL METHOD AND INITIAL CONDITIONS}

The numerical features of the LES code LESTUF are described in references [15] and [16]. For convenience, we briefly delineate the most important features. LESTUF solves the Boussinesq-approximated Navier-Stokes equations in staggered and Cartesian coordinates with a finite differences method (central finite differences in space and Adams-Bashforth scheme in time). The dissipative character of the turbulent Smagorinsky closure model is reduced within the vortex cores with the help of the NaCoo-correction [17], which reduces the (numerically caused) decrease of tangential velocities and core radius growth. Hence NaCoo compensates the drawback of a limited resolution of the vortex cores, which must be accepted in order to account for a realistic description of the large-scale eddies of atmospheric turbulence. The vortex parameters of the two counterrotating Lamb-Oseen vortices with $r_{c} / b_{0}=0.064$ are set to represent a landing Airbus A340-300 with $b_{0}=47.1 \mathrm{~m}$ and $\Gamma_{0}=458 \mathrm{~m}^{2} / \mathrm{s}$. In order to account for exhaust jet and boundary layer turbulence entrained during roll-up an additional $\mathrm{rms}$ velocity with $\sigma=2 \mathrm{~m} / \mathrm{s}$ is added in the vortex core radius vicinity. The mean initial descent velocity is calculated according to the Biot-Savart law to $w_{0}=\Gamma /$ $\left(2 \pi b_{0}\right)=1.54 \mathrm{~m} / \mathrm{s}$ and hence the reference time scale $t_{0}=b_{0} / w_{0}=30.5 \mathrm{~s}$ [4]. In order to allow for the formation of Crow instability the chosen computational domain amounts to $\mathbf{L} / b_{0}=(8.5,5.4,5.4)^{T}$ with a uniform and equidistant grid resolution of $\Delta=1 \mathrm{~m}$ (total grid points $400 \times 256 \times 256$ ). Using an explicite scheme requires an adequate Courant number, which should be of order 0.25 in LESTUF. A constant time step of $\Delta t=0.01 \mathrm{~s}$ arises for all LES, leading to one day computation time per $t_{0}$ vortex life time on a NEC SX6 supercomputer. Circulation is calculated as an average of eleven circular cross-sections normalized by the initial root circulation $\Gamma_{0}$ according to

$$
\Gamma_{5-15}^{*}=\frac{1}{11} \sum_{r=5 \mathrm{~m}}^{15 \mathrm{~m}}\left(\int_{0}^{r} \omega \mathrm{d} \mathbf{A}\right) / \Gamma_{0}
$$

The definition of $\Gamma_{5-15}^{*}$ is chosen because first it is supposed to represent total circulation of large aircraft sufficiently $[\mathbf{1 1}, \mathbf{1 8}]$, second it is easy to obtain by post-processing of experimental Lidar data and third it is assumed to be proportional to resulting rolling coefficients for encountering aircraft and is hence a measure for the remaining risk potential [19]. For these reasons $\Gamma_{5-15}^{*}$ has become a standard reference value for wake vortex strength during the last 10 years.

The Lamb-Oseen vortices are superimposed to a pre-generated field of turbulent background flow with periodicity in all three directions, solenoidality, homogenity, and an inertial subrange with a spectral shape of $k^{-5 / 3}$ according to Kolmogorov. If the characteristic length of the vortex pair, $b_{0}$, is situated well within the inertial subrange, turbulence intensity can be characterized by the eddy dissipation rate $\epsilon$ alone [20]. It is normalized based on wake vortex parameters according to $\epsilon^{*}=\left(\epsilon b_{0}\right)^{1 / 3} / w_{0}$ [11]. Values of 
$\epsilon^{*}=[0.01,0.05,0.23]$ are used to represent low, moderate, and strong turbulence, respectively.

Stable temperature stratification is added using a constant temperature gradient which yields normalized Brunt-Väisälä freqencies $N^{*}=t_{0} N=t_{0}$ $\sqrt{g / \theta_{0} \partial \theta / \partial z}$ of $N^{*}=[0,0.35,1]$ representing neutral, moderately stable, and very stable conditions, respectively.

Because of limited computing resources, however, it is not possible to generate both a fully developed turbulence field with a sufficient number of large turbulent eddies of the order of one $b_{0}$ and a sufficient resolution of the tight vortex cores. A quantitative measure for the mean eddy size is provided by the integral turbulent length scale which is defined as

$$
L_{t}=\frac{1}{4 v_{r m s}^{2}} \int_{\mathbf{k}=0}^{\infty} \frac{\hat{E}(\mathbf{k})}{\mathbf{k}} \mathrm{d} \mathbf{k}, \quad \mathbf{k}=\left|k_{1}, k_{2}, k_{3}\right|
$$

where $\hat{E}(\mathbf{k})$ represents the spectrum of the turbulent kinetic energy density in Fourier space.

To investigate the impact of $L_{t} / b_{0}$ on vortex evolution, simulations with varying length scales of $L_{t} / b_{0}=[0.16,0.41,0.66,0.85]$ were performed for the case $\epsilon^{*}=0.23$ and $N^{*}=0$. To achieve larger turbulence length scales the domain size was extended to $\mathbf{L} / b_{0}=(8.5,8.2,8.2)^{T}$ (mesh resolution unchanged) which is nearly cubic, so here the above definition is very well suited. However, in very stable conditions vertical velocity correlations are hampered hence these become much smaller than those in horizontal direction. If the influence of temperature stratification dominates the turbulent velocity field, it is differentiated between horizontal $\left(L_{t,(x, y)}\right)$ and vertical $\left(L_{t, z}\right)$ integral length scales.

\section{QUANTIFICATION OF VORTEX TUBE DEFORMATION}

\subsection{Vortex core line}

In order to quantify the vortex tube deformation via the estimation of curvature radii of vortex segments the vortex core line has to be detected precisely in three-dimensional (3D) space. Therefore a couple of different strategies are known from literature. Candidate criteria for the determination of the vortex centre are based on streamlines, maximum absolute vorticity, or minimum pressure. However, these methods do not always succeed in complex flowfields, so more sophisticated models have been developed [21]. Reference [22] suggests that complex eigenvalues of the velocity gradient tensor indicate the position of a vortex. A similar method was used by Jeong and Hussain [21] based on eigenvalues of the symmetric and the antisymmetric parts of the velocity gradient tensor. The proposed $\lambda_{2}$-definition corresponds to the pressure minimum in a plane when contributions of unsteady irrotational straining (shear vorticity cannot adulterate resulting vortex positions) and viscous terms are discarded. Alternative methods employ two parameters, a first one in a predictor step and a second one in a corrector step. Reference [23] used a combination of swirl number and pressure minimum, whereas in reference $[\mathbf{2 4}]$ a combination of vorticity and pressure was taken.

The method suggested here is in line with the latter approach, which benefits from a relatively simple implementation and nevertheless provides robust and reliable results. The vorticity vector is used to determine the direction of the vortex tube in the predictor step. For the correction step, several possible parameters have been tested to determine the vortex centre. It turned out that the determination of the pressure minimum works more reliably than absolute vorticity maxima, $\lambda_{2}$ minima $[\mathbf{2 1}]$, or a recently tested streamline method which calculates vortex centers from streamline curvature $[\mathbf{1 7}, \mathbf{2 5}]$. The proposed method is able to identify the vortex core line also under difficult conditions like progressed vortex decay with largely eroded vortex cores or the process of vortex linking.

The algorithm proceeds in four steps as shown in Fig. 1.

1. Determination of a starting point $\mathbf{x}_{0} \in G$ with $G$ being the set of all gridpoints. Since the pressure gradient is well established in fully rolled-up aircraft wake vortices, the initial positions $y_{0}$ and $z_{0}$ are found by determining the local pressure minimum in a suitably chosen initial grid plane $x=x_{0}$.

2. Around $\mathbf{x}_{i}$ the $n^{3}$ surrounding vorticity vectors $\omega$ are summed (with $n$ being any uneven integer). If necessary each single vector is interpolated within the eight surrounding grid points. The predicted position $\tilde{\mathbf{x}}_{i+1}$ is the sum of the preceding position $\mathbf{x}_{i}$ and the product of a predefined length $d$ and normalized vorticity $\omega_{i} / \omega_{i}$. It is-

$$
\tilde{\mathbf{x}}_{i+1}=\mathbf{x}_{i}+\frac{d \cdot \sum_{\mathbf{j} \in J_{i}}\left(\omega_{x}(\mathbf{j}), \omega_{y}(\mathbf{j}), \omega_{z}(\mathbf{j})\right)^{T}}{\sqrt{\left(\sum_{\mathbf{j} \in J_{i}} \omega_{x}(\mathbf{j})\right)^{2}+\left(\sum_{\mathbf{j} \in J_{i}} \omega_{y}(\mathbf{j})\right)^{2}+\left(\sum_{\mathbf{j} \in J_{i}} \omega_{z}(\mathbf{j})\right)^{2}}}
$$

with

$$
\begin{aligned}
J_{i}= & \left\{\mathbf{x}_{i}+\Delta(\xi, \psi, \zeta), \quad \frac{1-n}{2} \leqslant \xi, \psi, \zeta \leqslant \frac{n-1}{2}\right. \\
& \xi, \psi, \zeta \in \mathbb{Z}, \quad[(n+1) / 2] \in \mathbb{N}\}
\end{aligned}
$$

3. Around the predicted position $\tilde{\mathbf{x}}_{i+1}$ a cube with side length $c$ is defined, wherein the final position $\mathbf{x}_{i+1}$ is 
determined. Employing a centre-of-gravity method yields

$$
\mathbf{x}_{i+1}=\tilde{\mathbf{x}}_{i+1}+\frac{\sum_{\mathbf{m} \in M_{i}} \mathbf{m} p(\mathbf{m})}{\sum_{\mathbf{m} \in M_{i}} p(\mathbf{m})}
$$

with pressure $p(\mathbf{m})$ and

$$
M_{i}=\left\{\mathbf{m} \mid\left\|\mathbf{m}-\tilde{\mathbf{x}}_{i+1}\right\|_{\infty} \leqslant c\right\}
$$

4. Repetition from step (b) until either a vortex ring is completed or both parallel vortex tubes have been found throughout the computational domain.

The parameters $n, c$, and $d$ feature a small sensitivity to $n$ and a moderate sensitivity to $c$ and $d$. Good results were obtained with $n=5, c=10 \mathrm{~m}$, and $d=3 \mathrm{~m}$.

\subsection{Vortex curvature}

The curvature of the vortex core line is supposed to be quasi-2D within segments of a given size, which means that the local vortex deformation can be represented by the radius of a circle in one plane. For the development of the sinusoidal Crow instability prior to linking this assumption is very well suited [5]. After linking vortex rings develop which typically consist of almost planar core line segments as well. This is demonstrated in Fig. 2 in (a) top view and (b) side view. Circulation is grey-scale coded, which helps to identify corresponding vortex ranges in both views.

Extensive testing showed that vortex segments of length $1.5 b_{0}$ are well suited for both an idealised test case with sinusoidal vortex lines and a LES of a wake vortex pair evolution in a turbulent environment. Additionally, this length fits well to the idea of having segment lengths in aircraft relevant scales. Convergence was found in so far that larger vortex segments did not change the results noticeably, whereas smaller segments resulted in curvature radii, which are overly sensitive to small turbulence induced pertubations [26]. Additionally, those are irrelevant regarding encounters with following aircraft.

The method can be summarized by three steps for each point of the vortex core line, which are presented graphically in Fig. 3.

1. Determination of a vortex segment with an approximated length of $1.5 b_{0}$ (shown in black).

2. Specified by its normal, a plane $E$ is identified with minimum quadratic deviation distance of all associated vortex core positions. Then a first guess of the centre of the desired curvature circle $M_{0} \in E$ is determined which is the basis for a star-shaped search pattern of points $P(r, \phi) \in E$ varying $r$ and $\phi$ in the range of $0<r<5 \quad b_{0}, \quad 0^{\circ}<\phi<360^{\circ}$, $\Delta \phi=5.6^{\circ}$ (Fig. 3(b)). In order to save computational costs, the search pattern of this run is thinned out in the vicinity of $M_{0}$ which yields node intervals of comparable size.

3 . For each $P(r, \phi)$ circles with varying potential curvature radii $\left(0<\tilde{r}_{\kappa}<5 b_{0}\right)$ are computed and their distance $d$ (vortex line, $\tilde{r}_{\kappa}$ ) from the associated vortex points is calculated. The circle centre $M_{1}=P\left(r_{1}, \phi_{1}\right)$ achieving minimum quadratic deviations $\sum d^{2}$ from the vortex segment provides a preliminary curvature radius $r_{\kappa}=\tilde{r}_{\kappa}\left(r_{1}, \phi_{1}\right)$. This

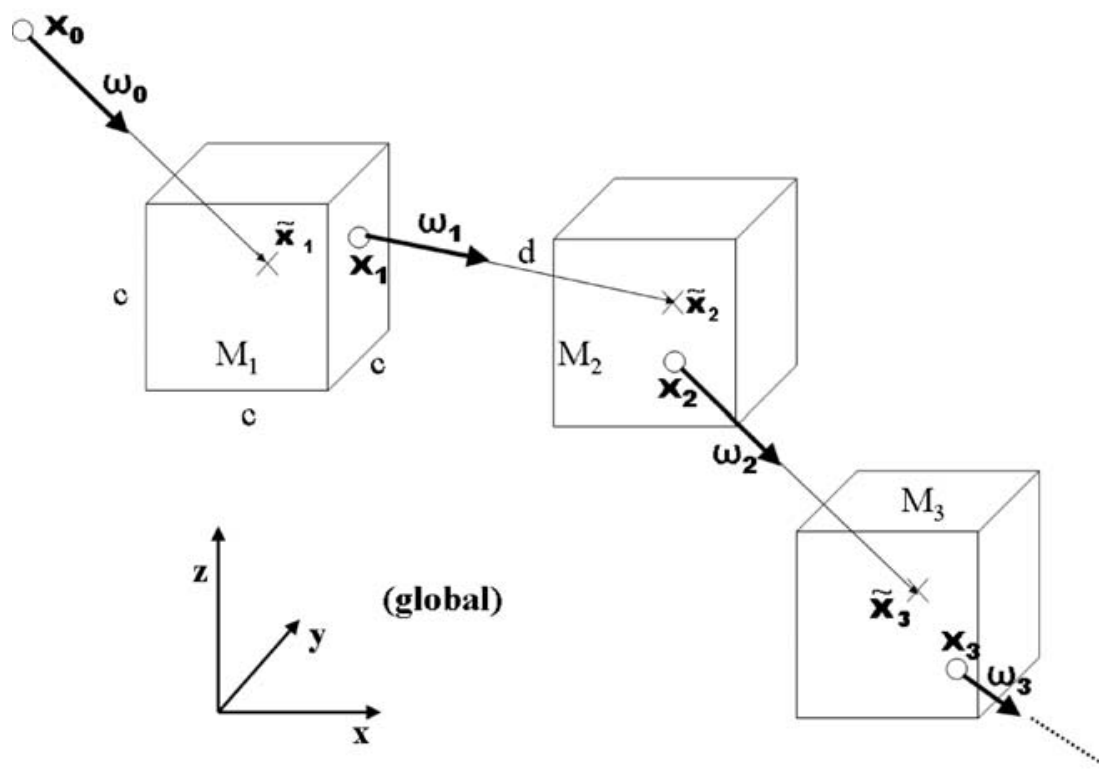

Fig. 1 Scheme of the predictor-corrector algorithm for the identification of the vortex core line 

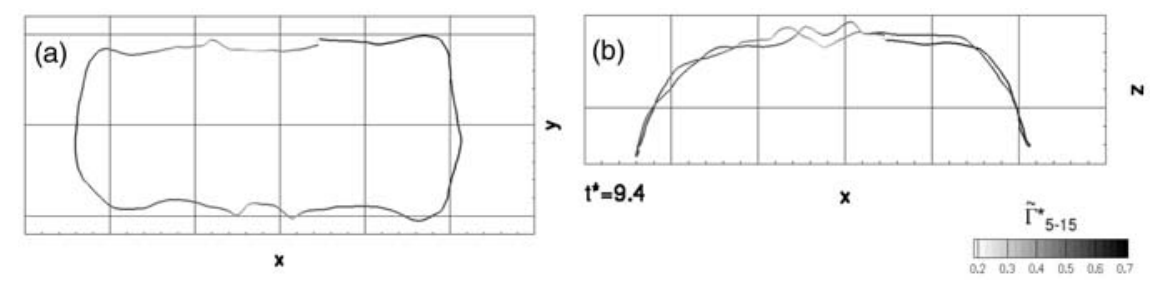

Fig. 2 Validity of assumption of piecewise planarity in vortex ring regime in (a) top view and (b) side view. Circulation along the vortex core line is grey-scaled $\left(N^{*}=0, \epsilon^{*}=0.01\right)$

(a)

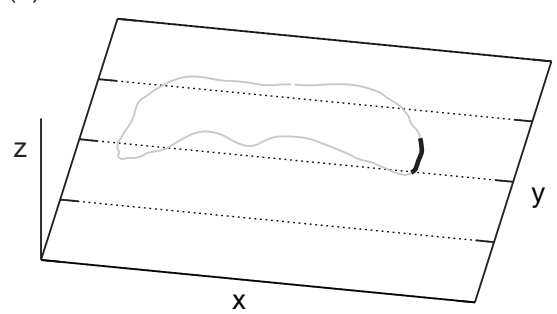

(c)

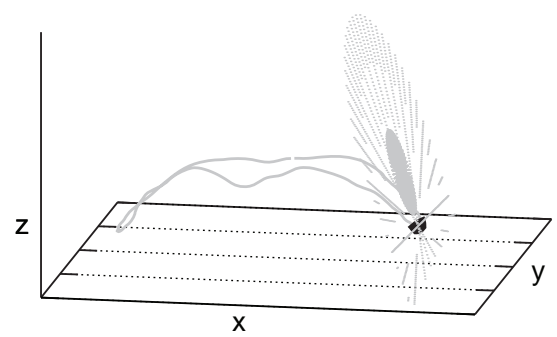

(b)

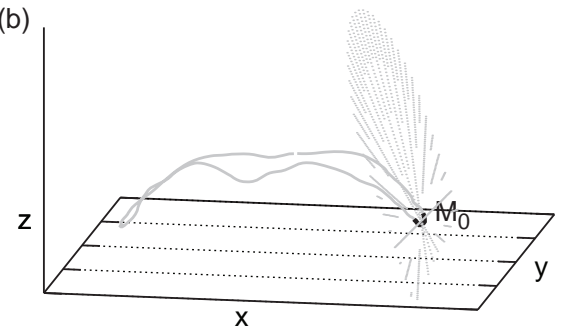

(d)

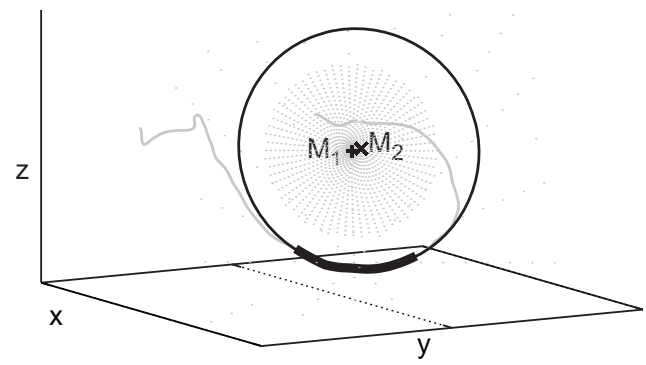

Fig. 3 Determination of vortex curvature for one vortex segment: (a) Vortex ring with black vortex segment; (b) Determination of plane $E$ which contains vortex track points best. Addition of coarse star-shaped search pattern around initial point $M_{0}$; (c) Addition of fine search pattern centred at preliminary curvature radius origin $M_{1}$; (d) Final detection of curvature circle with radius $r_{\mathrm{K}}=1.4 b_{0}$

procedure is repeated with finer resolution now starting at $M_{1}$ until $M_{2}$ is found (Figs 3(c) and (d)).

In the presented study the algorithm is adjusted such that it allows maximum curvature radii of $r_{\kappa} \approx 6 b_{0}$. For all cases, the mean difference between the actual 3D vortex core positions and the estimated circle segments within the chosen $2 \mathrm{D}$ planes is at most $8-10$ percent for $N^{*}=1$ and smaller than 3 per cent for neutral stratification.

\section{RESULTS}

\subsection{Circulation evolution}

For each of the nine investigated variations of turbulent dissipation rate $\epsilon^{*}$ and Brunt-Väisälä frequency
$N^{*}$ Fig. 4 shows the evolution of circulation, $\Gamma_{5-15}^{*}$, against normalized time $\left(t^{*}=t / t_{0}\right)$ with two curves: lines represent circulation determined in planes perpendicular to the flight direction $\left(\Gamma_{5-15}^{*}\right)$, and symbols represent circulation determined perpendicular to the local vortex core line $\left(\tilde{\Gamma}_{5-15}^{*}\right)$. The instant when both curves separate indicates the time of vortex linking. Starting from that time a non-negligible amount of vorticity is oriented in span direction. If circulation drops below a certain value (here 10 per cent of its initial value), it is assumed that the requirements for the existence of a vortex, a coherent vortex structure and corresponding circulation, are not fulfilled any more. From that point the vortices are said to have ceased. Some simulations without temperature stratification had to be stopped before that state because the resulting vortex rings broadened such that they exceeded the computational domain of width 


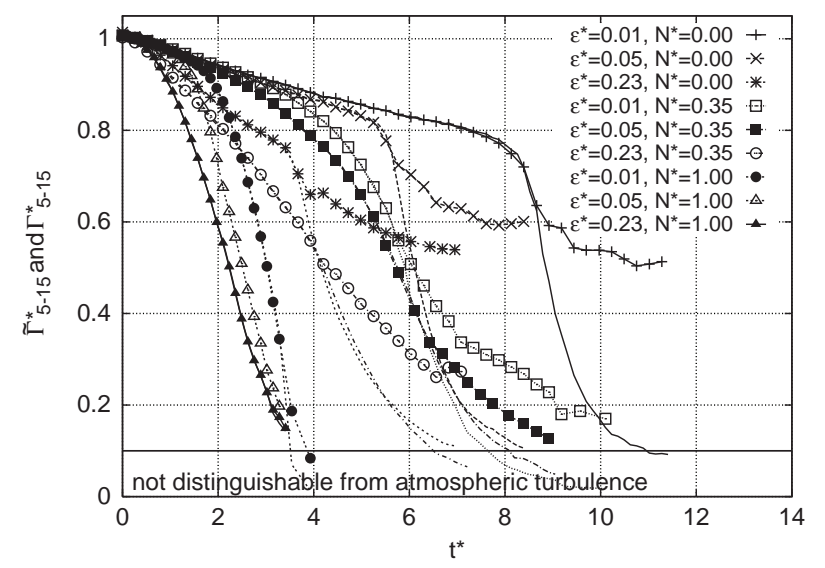

Fig. 4 Circulation evolution for different temperature stratification and ambient turbulence

$L_{y} / b_{0}=5.4$. More details regarding ring formation and evolution are described in the next section.

Figure 4 indicates that at least one of the parameters $N^{*}$ and $\epsilon^{*}$ must be strong in order to accelerate vortex decay. However, the respective mechanisms differ. Since the influence of temperature stratification (namely the production of baroclinic vorticity) is well documented (e.g. references [18, 27]), this section focusses on the impact of turbulence. The twophase circulation decay pattern found in reference [11] is appropriate if circulation is calculated perpendicular to flight direction. However, the consideration of circulation perpendicular to the vortex line allows deeper insights into the real physical development from the initially straight vortices to the formation of vortex rings. For neutral and weakly stratified conditions $\tilde{\Gamma}_{5-15}^{*}$ shows a three-phase decay, which can be assigned to the established terms 'diffusion phase' for the first phase, a short phase of rapid decay and a third and new phase of gradual decay comparable to the first one which is termed 'ring diffusion phase'.

It was found that the rapid decay phase consists of two mechanisms that are responsible for a quicker decay of circulation. First, when both vortices approach small-scale instabilities occur which require $r_{c} / b \geqslant 0.2$. This transition from large-scale to small-scale deformation accelerates turbulent decay [28]. Second, the integration of $\tilde{\Gamma}_{5-15}^{*}$ includes counter-rotating vorticity of the adjacent vortex, which modifies the velocity profile. In literature this is sometimes called 'viscous cancellation' [14] or 'annihilation of vorticity' [13]. This process emanates centrally between the vortices, and so both vortex tubes break up which initiates the reconnection process. After a short time, the four remaining tips of both broken vortex tubes are located such that initially adjacent vortices are much closer than initially

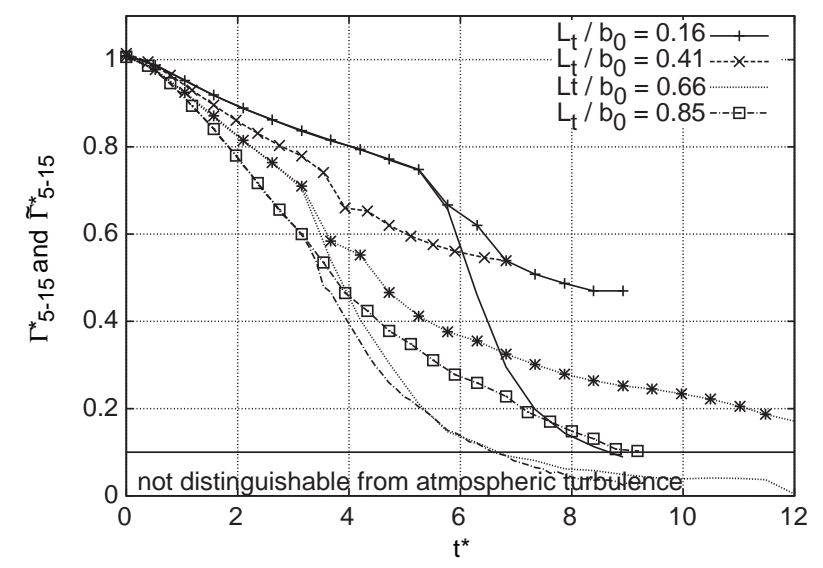

Fig. 5 Circulation evolution for different turbulent integral length scales and $\epsilon^{*}=0.23$ and $N^{*}=0$

connected vortices, leading to a reconnection across the $x$-axis. If this happens simultaneously at two neighbouring approach zones a vortex ring has formed.

Results of the simulation series with varying turbulent length scales are shown in Fig. 5. Again, symbols indicate $\tilde{\Gamma}_{5-15}^{*}$ and lines represent $\Gamma_{5-15}^{*}$. Due to the wider computational domains used for the cases with $L_{t} / b_{0} \geqslant 0.66$, these simulations could be carried out until the vortices were hardly recognizable any more. Except for $L_{t} / b_{0}=0.16$ the onset of rapid decay occurs at similar instants of time, but the corresponding circulation evolution in the diffusion phase differs strongly. With increasing $L_{t} / b_{0}$ the circulation decay in the diffusion phase speeds up and, as a consequence, the three-phase decay is more and more concealed.

For integral length scales exceeding one initial vortex spacing the influence of $L_{t}$ can be neglected as a parameter for vortex decay characterization $[\mathbf{2 0}$, 29]. However, if $L_{t} / b_{0}<1$ it should be considered together with $\epsilon^{*}$. For example, in the atmospheric surface layer $L_{t}$ depends on the distance to the ground and can reach small values in ground proximity. There, wake vortex evolution is crucial because many encounters occur close to the runways at altitudes below $100 \mathrm{~m}$ where the wake vortices can not descend below the flight path [30]. In field experiments it has been observed that wake vortex decay in ground proximity is only very weakly dependent on the eddy dissipation rate $[\mathbf{3 0}, \mathbf{3 1}]$. The described sensitivity on $L_{t} / b_{0}$ suggests that this is related to the reduced turbulence length scales in the surface layer. Consequently, vortex decay mechanisms at the ground are mainly controlled by the interaction of the wake vortices with the secondary vortices detaching from the boundary layer [30]. 

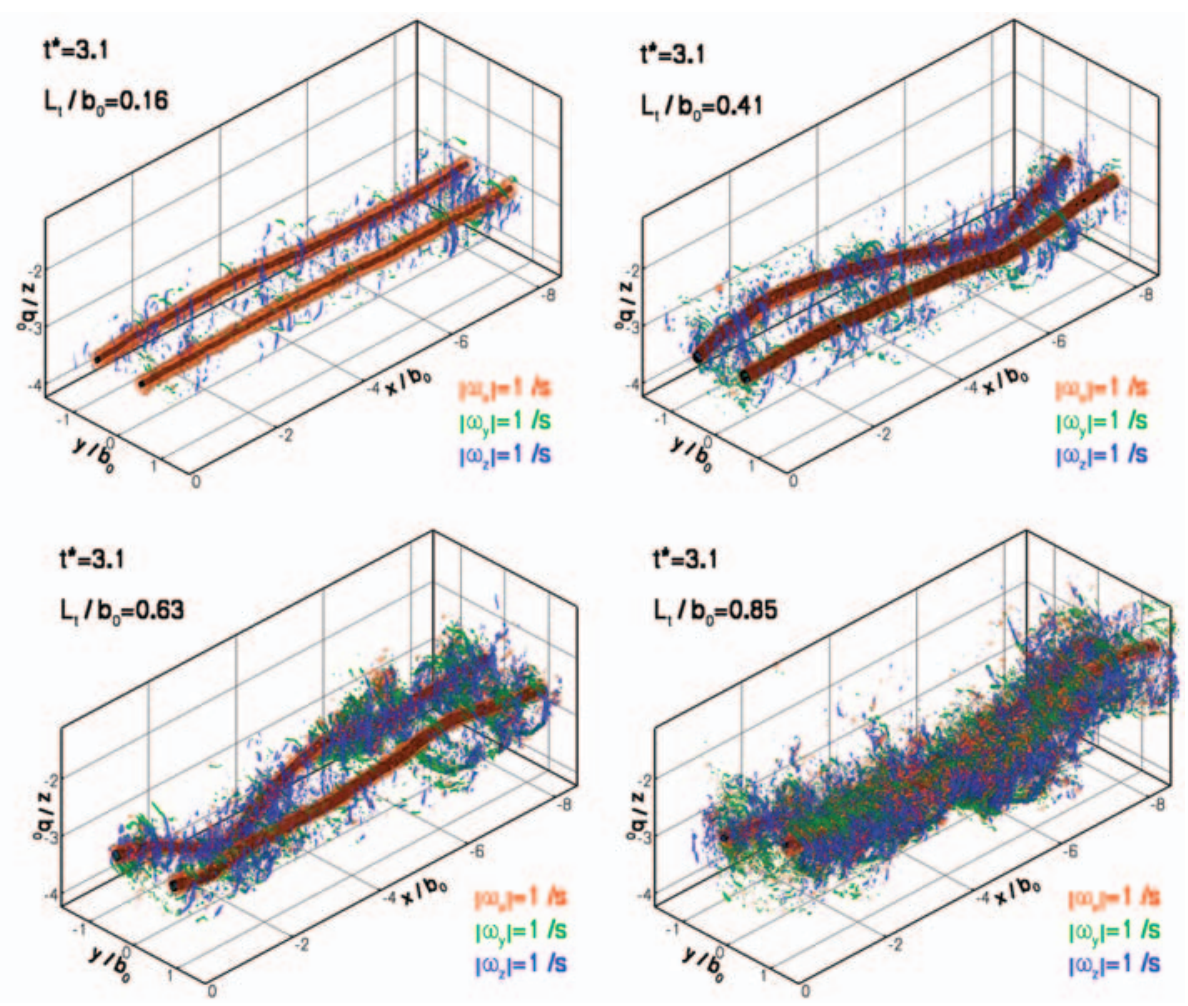

Fig. 6 Secondary vorticity production during diffusion phase for varying turbulent length scales with $N^{*}=0$ and $\epsilon^{*}=0.23$ at $t^{*}=3.1$

Figure 6 shows the evolution of secondary vorticity for varying length scales prior to the rapid decay phase at $t^{*}=3.1$. The solid black lines represent pressure isosurfaces as an indicator for the vortex core, surrounded by all three vorticity components with a constant magnitude of $1 \mathrm{~s}^{-1}$. Because the pressure isosurfaces of $L_{t} / b_{0}=0.16$ are thinner, it can be concluded that the core is still more compact than in the other LES. $\omega_{x}$ is situated around the pressure isosurfaces only, whereas $\omega_{y}$ and $\omega_{z}$ are mixed and appear anywhere within and around Kelvins vortex oval. The series shows that deformation and the total amount of surrounding secondary vorticity increases with $L_{t}$ for $L_{t}<b_{0}$.

The relevant production terms for secondary vorticity are given by the vorticity formulation of the impulse equation (neglecting viscous and compressible effects)

$$
\frac{\partial \boldsymbol{\omega}}{\partial t}=-(\mathbf{u} \cdot \nabla) \boldsymbol{\omega}+(\boldsymbol{\omega} \cdot \nabla) \mathbf{u}+\frac{1}{\rho^{2}} \nabla \rho \times \nabla p
$$

The RHS terms can be denoted as advection term, tilting and stretching term, and baroclinic vorticity production term. In stably stratified cases the last term produces baroclinic vorticity which can be neglected here because $N^{*}=0$. Nevertheless any spatial gradient of velocity or vorticity is sufficient to amplify the production of secondary vorticity, as described in reference [32]. It is supposed that environmental turbulent eddies interact with the primary vortices resulting in an energy transfer from the vortex pair to secondary vorticity structures. This is achieved by strechting and tilting mechanisms which are stronger if more eddy energy is included in those modes of comparable size to the vortex spacing. For $L_{t}>b_{0}$ turbulent eddies of sizes that are too large to be stretched by the wake vortices are added. Those are excluded from the energy transfer mechanism.

\subsection{Circulation evolution in the ring diffusion phase}

The sinusoidal vortex topology caused by the Crow instability is driven by mutual velocity induction according to the law of Biot-Savart

$$
\mathbf{u}=\frac{\Gamma}{4 \pi} \int \frac{\mathrm{d} \omega \times \mathbf{1}}{|\mathbf{1}|^{3}}
$$

with $\mathbf{l}$ being the vector from the position of $\mathbf{u}$ to the position of the vorticity segment $\mathrm{d} \omega$. This holds also for the subsequent vortex evolution, in particular, during the early ring diffusion phase. Here vortex dynamics cause both quick deformations of the overall topology of the vortex ring and changes of the local 
tangential velocity profiles corresponding to changes of the vortex core radii, $r_{c}$. According to equation (1) $\tilde{\Gamma}_{5-15}^{*}$ is sensitive to $r_{c}$ because it averages circulation values between $5 \mathrm{~m}$ and $15 \mathrm{~m}$ where the vortex velocities strongly depend on $r_{c}$. In inviscid flow increasing values of $r_{c}$ cause decreasing $\tilde{\Gamma}_{5-15}^{*}$ values in contrast to $\Gamma_{0}$ which remains constant according to the first theorem of Helmholtz.

Hence, in order to understand the evolution of $\tilde{\Gamma}_{5-15}^{*}$ and $\Gamma_{5-15}^{*}$ it is worthwhile to consider the vortex core radius evolution. Figure 7 shows the ranges of $r_{c}$ and $\tilde{\Gamma}_{5-15}^{*}$ for the case $\left[N^{*}=0, \epsilon^{*}=0.23\right.$ ] before $\left(t^{*}=3.7\right)$, during $\left(t^{*}=3.8\right)$ and after linking $\left(t^{*} \geqslant 5.0\right)$. Solid curves representing the core radius are shown at their true positions, whereas the dashdotted circulation curves are slightly shifted for better readability.

Around $t^{*}=5.0$ the core radii $r_{c}$ and the circulation values $\tilde{\Gamma}_{5-15}^{*}$ are negatively correlated along the vortex ring. In flight direction maximum core radii are observed corresponding to low values of $\tilde{\Gamma}_{5-15}^{*}$. Initially the vortex ring is long (in flight direction) and slender (in spanwise direction), but then it shortens and broadens with time. Both shortening and broadening are directly related to mutual velocity induction, which is most pronounced if cooperating vortex segments are strong and close together. Figure 2(b) shows almost vertically oriented and pairwise vortex segments whose mutually induced velocities point inwards. Hence the downward velocity of the initially parallel vortex pair at $t^{*}=0$ is partly replaced by a velocity component which compresses the vortex ring.

However, these two pairs of almost vertically oriented vortex segments do not only induce the compression of the vortex rings in flight direction but also its broadening in spanwise direction: The pairings of the vertically inclined vortex segments on the right and on the left yield an outward directed velocity in $y$-direction (due to the cross product of equation (6)). Consequently broadening is most pronounced close to the vertically inclined vortex segments (resulting in a bone-like structure in the $(x, y)$-plane). This effect is small in the early ring diffusion phase when the corresponding vortex segments are relatively far away from each other, but increases as the vortex spacing decreases. This results in an augmenting vortex width with an exponential character, see Fig. 8.

Figure 8 depicts the maximum vortex extension in flight direction $(\Delta x)$ and the maximum spanwise separation distance $(\Delta y)$ for the nine simulations with varying $\epsilon^{*}$ and $N^{*}$. Except for the LES with $\left[N^{*}=1\right.$, $\left.\epsilon^{*}=0.05\right]$ all vortex pairs did link, followed by a more or less distinctive compression mechanism of the vortex ring in flight direction and broadening in spanwise direction. For most cases the lateral vortex extensions exceed the axial extensions at the end of the respective simulations.
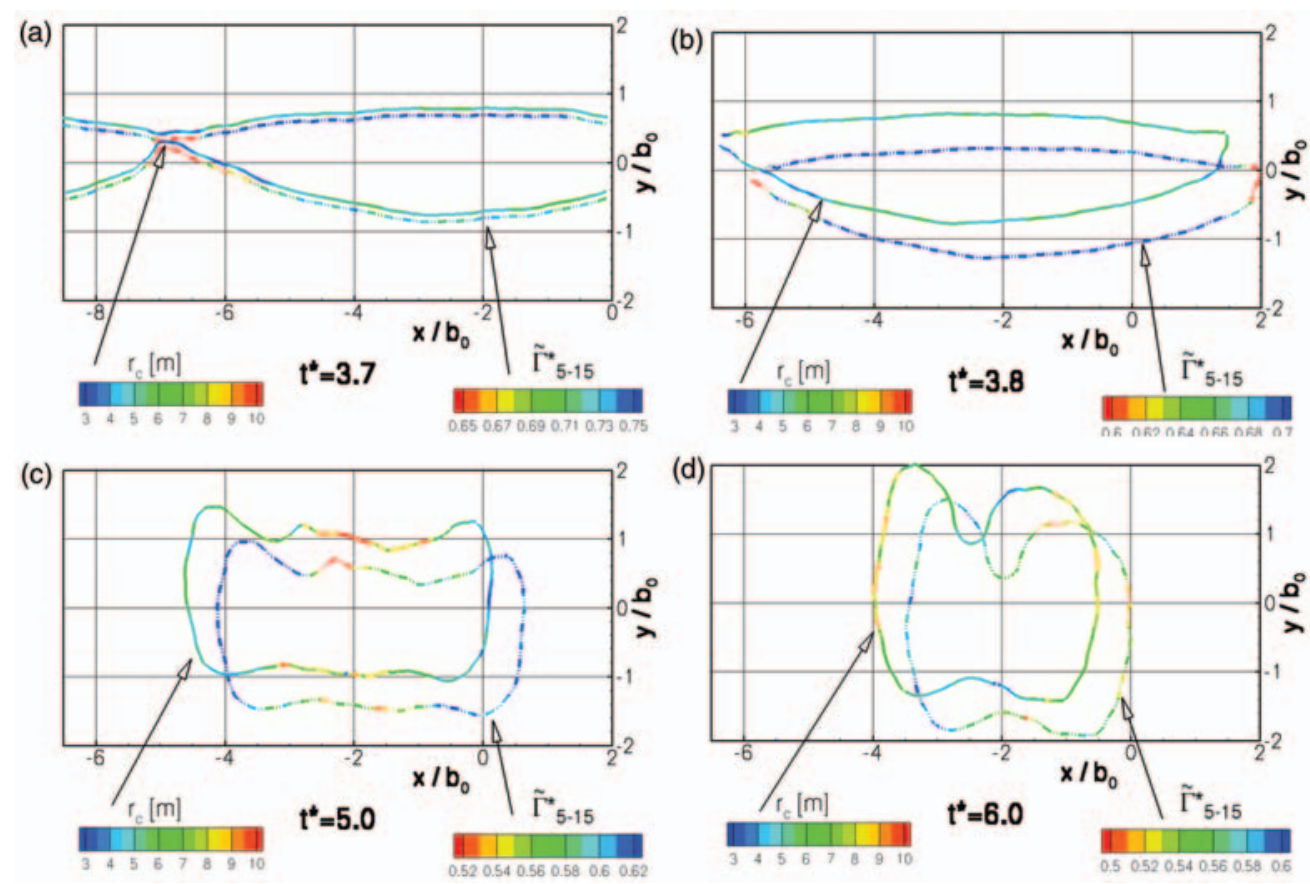

Fig. 7 Core radius evolution (solid line, true position) and circulation evolution (dot-dashed line, slightly displaced position) at (a) $t^{*}=3.7$, (b) $t^{*}=3.8$, (c) $t^{*}=5.0$, and (d) $t^{*}=6.0$ in turbulent atmospheric conditions $\left[N^{*}=0, \epsilon^{*}=0.23\right]$ 
In summary, 3D mutual velocity induction causes the vortex rings to shorten and broaden. Simultaneously, mutual velocity induction stretches parts of the spanwise oriented vortex segments and it squeezes parts of the vortex segments oriented in flight direction. These stretching and squeezing mechanisms are inversely affecting the vortex core size and radii-averaged circulation, $\tilde{\Gamma}_{5-15}^{*}$.

There is also another effect influencing core radii and circulation. The vortex linking process perturbs the pressure minimum on the vortex axis inducing pressure waves and corresponding axial velocities propagating from the disturbance along the vortex lines $[\mathbf{3 3}, \mathbf{3 4}]$. Eventually, the generated waves collide midway between the linking points. The collisions cause a sudden radial expansion of the vortices corresponding to a loss of $\tilde{\Gamma}_{5-15}^{*}$ (Fig. $7(\mathrm{c})$ ).

\subsection{Vortex curvature evolution}

In Fig. 9 probability distributions of curvature radii are shown before linking $\left(t^{*}=3.7\right)$, at linking $\left(t^{*}=3.8\right)$, at the beginning of the ring diffusion phase $\left(t^{*}=5.0\right)$ and during ring compression $\left(t^{*}=6.0\right)$, respectively. The bin size of the curvature radii is $0.1 b_{0}$, the environmental conditions are $N^{*}=0$ and $\epsilon^{*}=0.23$ again.

Between $t^{*}=3.7$ and $t^{*}=3.8$ there is a significant increase of very small curvature radii due to vortex linking. When the linking process is completed, $r_{\kappa} \approx b_{0}$ dominates. The continuous broadening of the vortex tubes in spanwise direction additionally deforms those segments in flight direction, resulting in the mentioned bone-like geometry in the $(x, y)$ plane (Fig. $7(\mathrm{~d})$ ). Hence the last large curvature radii

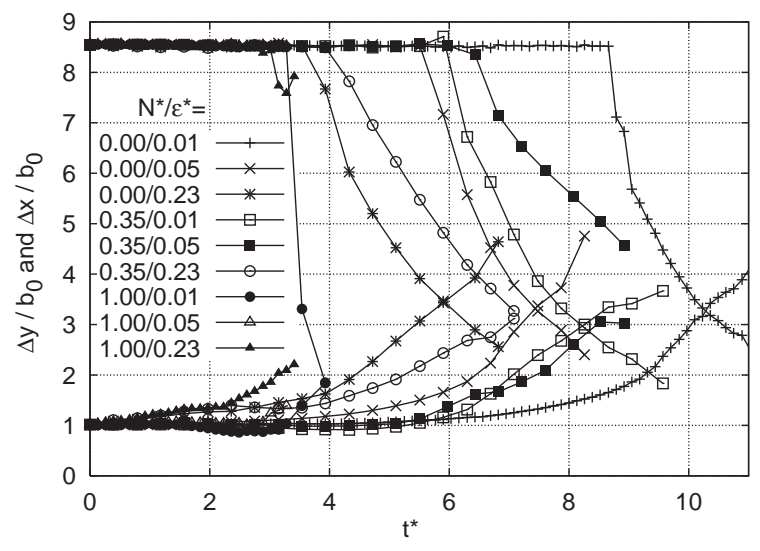

Fig. 8 Temporal evolution of maximum extension of the vortices in flight direction (starting at $\Delta x$ / $\left.b_{0}=8.6\right)$ and the separation in spanwise direction (starting at $\Delta y / b_{0}=1$ ) for the nine simulated cases disappear quickly. At $t^{*}=6.0$ the strong deformation tends to relax towards slightly higher and smaller values of $r_{\kappa}$. As can be seen in Figs 7 and 8, maximum extensions in both axial and lateral directions are almost identical at that time $\left(\Delta x \approx \Delta y \approx 3.5 b_{0}\right)$.

In order to analyse the influence of stratification Fig. 10 displays the curvature radii distributions for $\epsilon^{*}=0.23$ and $N^{*}=1$. Again, the plots refer to instants of time before linking $\left(t^{*}=2.1\right)$, at linking $\left(t^{*}=2.5\right)$, at the beginning of the ring diffusion phase $\left(t^{*}=2.9\right)$ and during ring compression $\left(t^{*}=3.3\right)$. In the strongly stratified case more small-scale deformations can be observed compared to neutrally stable conditions. The formation of a vortex ring does not change the deformation pattern as much as in Fig. 9 and the relaxation of curvature as seen in the preceeding case does not occur. A distribution maximum for $r_{\kappa} /$ $b_{0} \approx 0.5$ is found here which is caused by small-scale deformations due to baroclinic vorticity. It can be concluded that in analogy to circulation decay, vortex deformation increases if at least one of the conditions stable stratification or strong turbulence is fulfilled. Turbulence enforces the growth of the locally smooth and long-wave Crow pattern, which ends up in both strongly curved segments and nearly straight segments at the time of linking. In contrast, the production of small-scale baroclinic vorticity due to the adiabatically descending vortex oval enforces small-scale deformations to occur which results in a quicker decay in all stratification-dominated simulations.

Figure 11 displays curvature radius distributions at $t^{*}=[3.7,3.8,5.0,6.0]$ for $N^{*}=0, \epsilon^{*}=0.23$ and $L_{t} / b_{0}=0.85$. Compared to the case with smaller turbulent eddies, vortex deformation is more distinctive
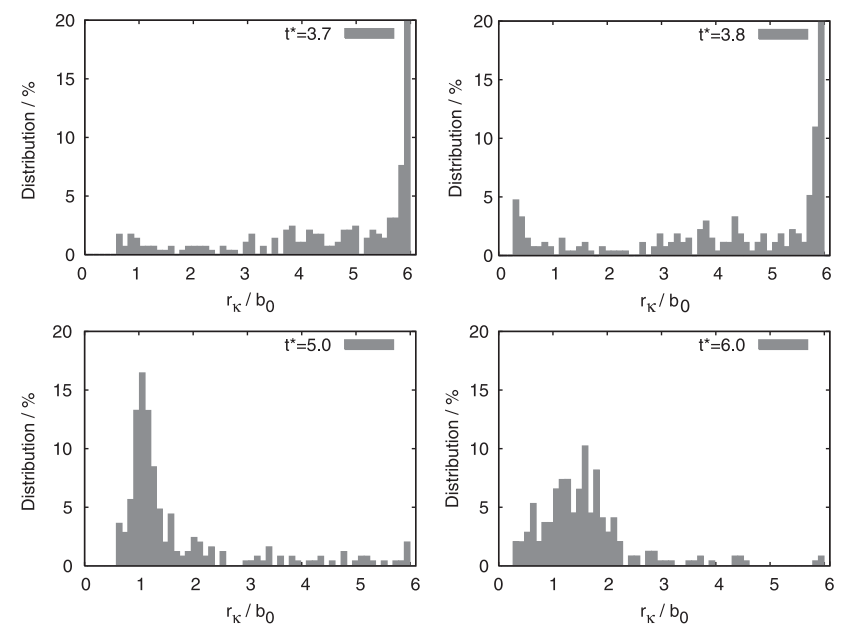

Fig. 9 Distribution of curvature radii during vortex ring formation with ambient conditions $N^{*}=0, \epsilon^{*}=0.23$ and $L_{t / b_{0}}=0.41$ 

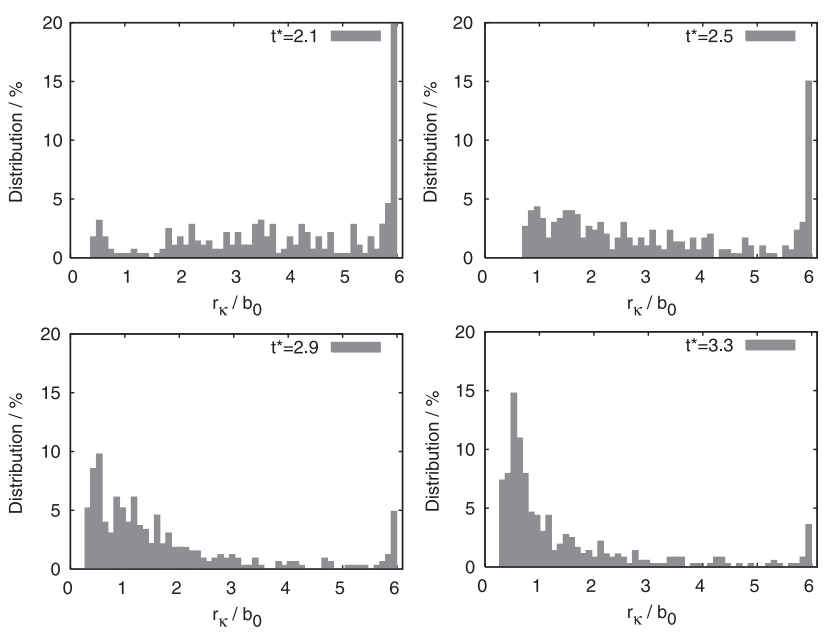

Fig. 10 Distribution of curvature radii during vortex ring formation with ambient conditions $N^{*}=1, \quad \epsilon^{*}=0.23, \quad L_{t,(x, y)} / b_{0} \approx 1.0$ and $L_{t, z} /$ $b_{0}=0.25$

in the diffusion phase. During that early stage the $r_{\mathrm{K}}$-distributions rather resemble the stably stratified case displayed in Fig. 10. After linking, both distributions (Figs 9 and 11) look similar.

Despite the wide range of meteorological conditions and the resulting vortex lifetimes and varying decay characteristics, the statistics of vortex curvature radii look quite similar for all the conducted LES. An inspection of the investigated cases reveals that the development of the curvature radii distributions is well correlated with circulation decay. Figure 12 displays joint probability density distributions (JPDDs) of curvature radii dependent on normalized circulation, which have been established from the post-processing of the nine LES.

Because the method to derive curvature radii employs a maximum of $r_{\kappa} / b_{0}=6$, vortex segments with $r_{\kappa} / b_{0}>5$ are considered to be straight in principle. Curvature radii $r_{\mathrm{\kappa}}$ are completely larger than $5 b_{0}$ only for $\Gamma_{5-15}^{*}>0.95$. Already at $\Gamma_{5-15}^{*}=0.8$ curvature radii below $r_{\kappa} / b_{0}=1$ show up. At $\Gamma_{5-15}^{*} \approx 0.6$ about 50 percent are still straight $\left(r_{\kappa} / b_{0}>5\right)$ and at $\Gamma_{5-15}^{*} \approx 0.25$ slightly more than 5 per cent of the vortices still feature curvature radii above $r_{\kappa} / b_{0}=5$. Possibly, a significant part of the straight vortex segments below $\Gamma_{5-15}^{*}=0.5$ are related to inflection points of the vortex center lines which can be approximated best by curve segments with large curvature radii. At a late stage of vortex evolution $\left(\Gamma_{5-15}^{*}<0.5\right)$ curvature radii accumulate somewhat below one initial vortex spacing. The established JPDD allows approximately predicting curvature radii distributions depending on the respective wake vortex circulation independent from the prevailing atmospheric conditions.
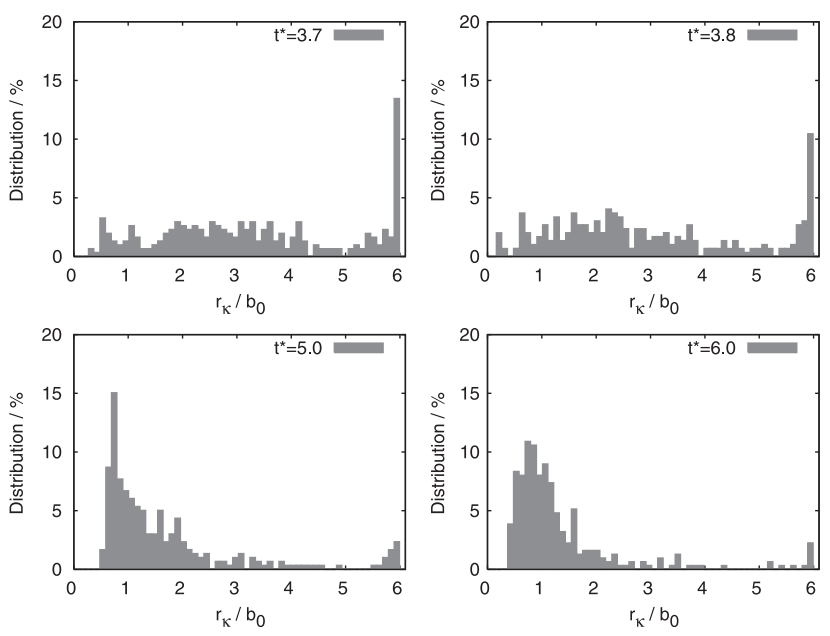

Fig. 11 Distribution of curvature radii during vortex ring formation with ambient conditions $N^{*}=0, \epsilon^{*}=0.23$ and $L_{t} / b_{0}=0.85$

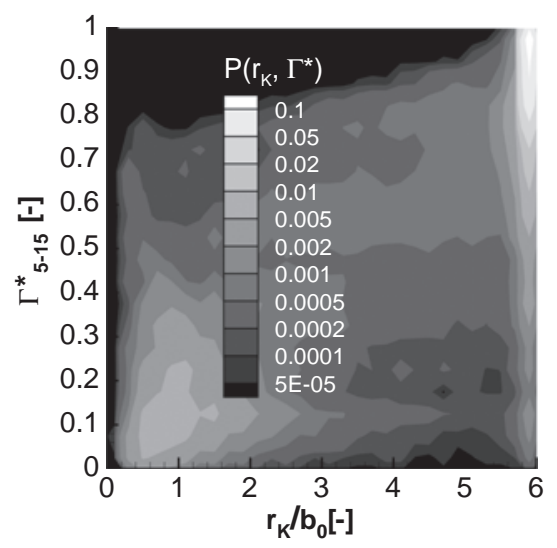

Fig. 12 Joint probability density distribution of curvature radii and circulation averaged for all parameter combinations of $N^{*}$ and $\epsilon^{*}$

\subsection{Vortex ring descent}

The simulations indicate that vortex rings may be very persistent. They continue descending as long as their circulation and the separation distance of the adjacent tubes remain, but the corresponding descent speed is obviously reduced compared to its initial value. An equation for the descent speed of a circular vortex ring has been derived theoretically by Saffman [35]. Based on experiments and numerical simulations reference [36] obtained an estimation for the mean- descent speed of recently formed elliptical vortex rings yielding $w_{\text {ring, } 0} \approx 0.6 w_{0}$.

Normalized mean vortex descent is shown in Fig. 13 for all parameter combinations of $N^{*}$ and $\epsilon^{*}$. 


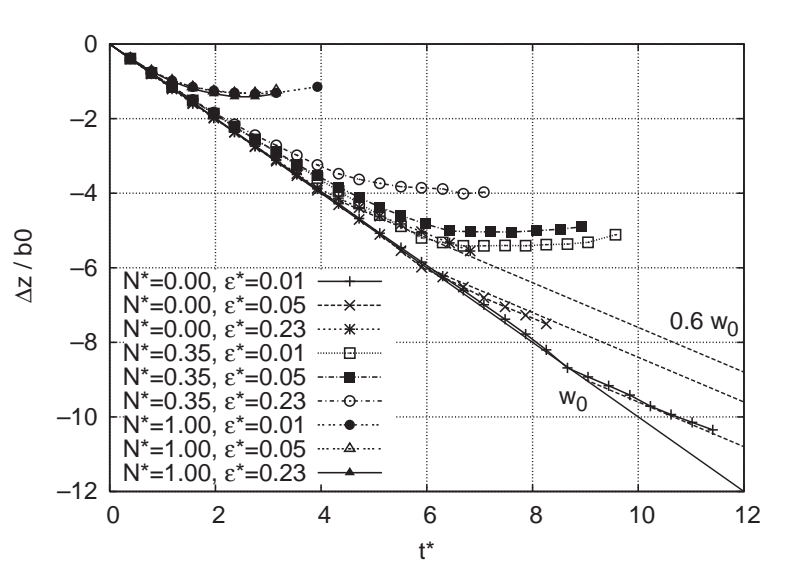

Fig. 13 Mean vortex descent for nine simulated cases

Initially the curves have a slope of -1 by definition. In the stably stratified cases the buoyancy force reduces the descent speed and may even cause a slight rebound for $N^{*}=1$. The maximum descent distances of $\quad \Delta z / b_{0} \approx-5.5 \quad\left(N^{*}=0.35\right) \quad$ and $\quad \Delta z / b_{0} \approx-1.5$ $\left(N^{*}=1\right)$ are confirmed numerically $[\mathbf{1 1}]$ and experimentally $[37,38]$. For $N^{*}=0$ the descent rate of $0.6 w_{0}$ after ring formation is well confirmed (Fig. 13). Figures 13 and 4 reveal that after the ring formation both $\Gamma_{5-15}^{*}$ and $w_{\text {ring }}$ merely decrease slowly for $N^{*}=0$. Notable maximum descent distances of more than $10 b_{0}$ are reached in the neutrally stratified calm atmosphere. It may be argued that the typical weak stable temperature stratification prevailing at cruise altitudes may prevent wake vortices from excessive descent distances reaching beyond adjacent flight levels.

\subsection{Comparison to field experiment data}

Because Lidar systems are typically scanning perpendicular to the flight direction they can (and do) confirm the onset of rapid decay (hence the first two phases), but the third ring-forming phase can only be derived indirectly. Therefore, also photographs are used to compare simulation results to real longlived vortex rings. In the EU-project AWIATOR [2] flights with an A340-300 were performed in order to investigate wake vortices in detail. Smoke generators were fixed at the wings for video recording, additionally Lidar measurements and meteorological measurements were carried out yielding circulation and position of the vortices and the corresponding atmospheric parameters wind speed, turbulent eddy dissipation rate, and temperature stratification.

The medium altitude A340 videos (FL-90, so-called 'Fly-ups') obtained by reference [39] revealed that in (a)

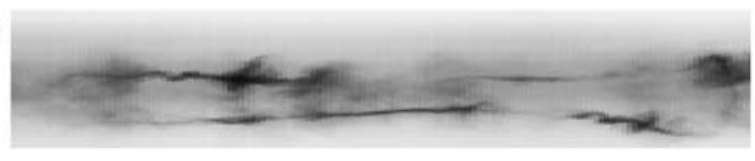

(b)

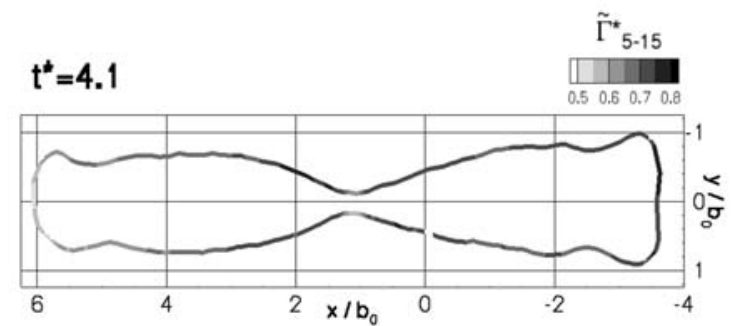

Fig. 14 (a) Stitched video data from AWIATOR 'Fly-up 4' (29.08.2003) ranging from $t^{*}=4.3$ to $t^{*}=4.6$ [39]. In b) the vortex state of a LES at $t^{*}=4.1$ including circulation is shown. Ambient conditions are approximately equal $\left(N^{*}=0.35\right.$, $\left.\epsilon^{*}=0.05\right)$

all flights the vortex tubes approached and affected each other and that in more than 50 percent of all observations the vortex tubes have linked at least once. Twice vortex rings with a total vortex ring length of $2 \lambda_{\text {Crow }}$ were observed. In Fig. 14 the meteorological conditions of the observation (measured at Cessna Citation observation aircraft at FL-75) and the simulation were $N^{*}=0.35$ and $\epsilon^{*}=0.05$. The respective vortex topologies look similar except that the approach in the centre of the double ring is further progressed in the LES. In particular, the additional broadening after linking is most pronounced at the top right corner. In Fig. 14(a) visibility may be related to circulation because tight vortices have a high optical thickness. So a similar evolution of vortex strength is found as well.

The LES prediction skill can be estimated more quantitatively employing wake vortex measurements applied on overflights made $1500 \mathrm{ft}$ above Tarbes airport [2]. Figure 15 shows the wake vortex parameters vertical position $\left(z / b_{0}\right)$, lateral spacing $\left(y / b_{0}\right)$ and circulation $\left(\Gamma^{*}\right)$ and the measured meteorological parameters headwind $\left(u^{*}\right)$, crosswind $\left(v^{*}\right)$, vertical wind $\left(w^{*}\right)$, RMS of turbulent velocity $\left(q^{*}\right)$, eddy dissipation rate $\left(\epsilon^{*}\right)$, and Brunt-Väisälä frequency $\left(N^{*}\right)$ of AWIATOR flight 2-04. Velocity parameters with superscipt '*' are normalized with the initial descent speed $w_{0}$. The effect of wind on the vertical position and on circulation is not considered in the LES. In principle, the vertical wind component $w^{*}$ can modify $z$ as $u^{*}$ can transport the vortex pair quicker into the Lidar measurement plane than anticipated by the LES. However, that requires at least $w^{*}>0.1$ or $u^{*}>0.1 V_{0}$ respectively, so both effects can be neglected in the presented case. 

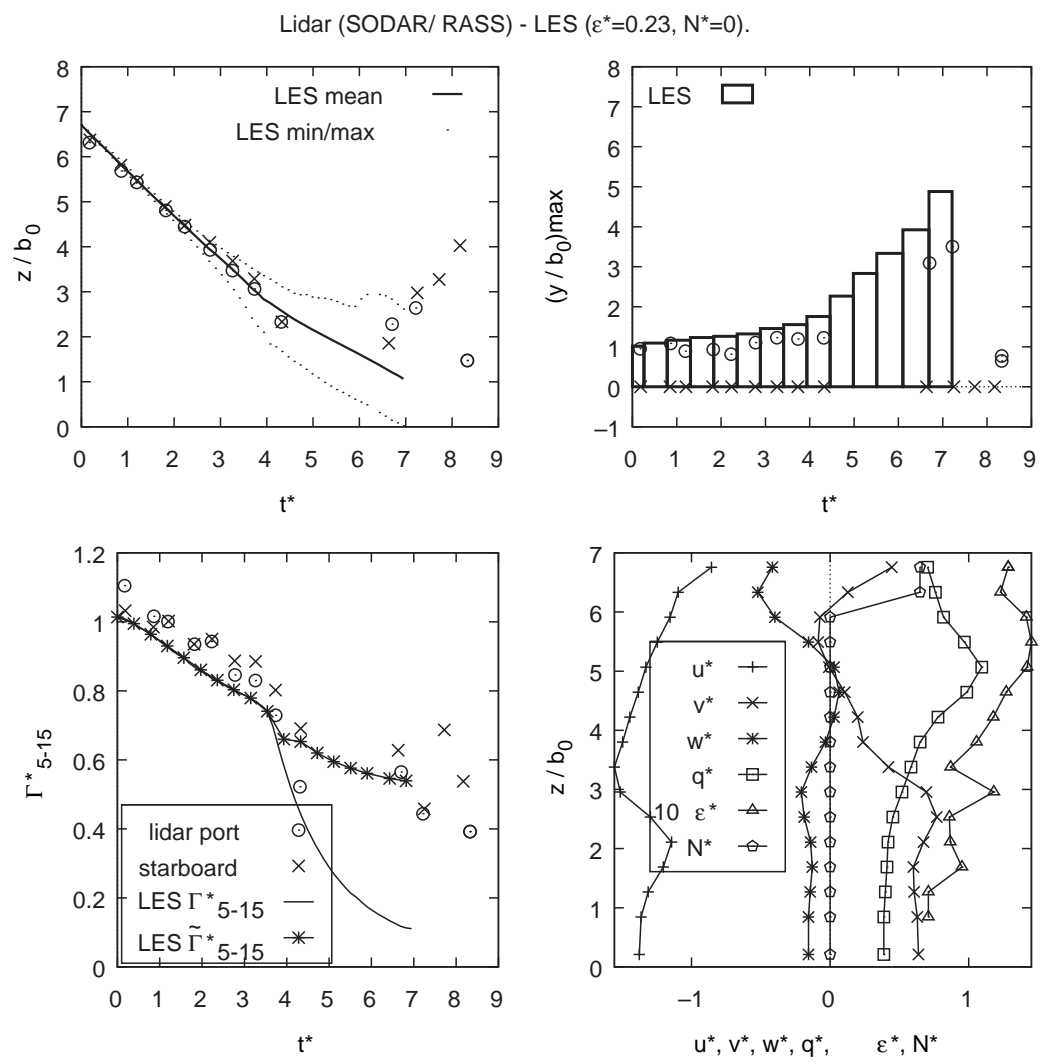

Fig. 15 Vertical position $\left(z / b_{0}\right)$, lateral spacing $\left(y / b_{0}\right)$, circulation $\left(\Gamma^{*}\right)$, and measured meteorological data of AWIATOR 'Fly-over 2-04' compared to LES results

The top left plot of vertical vortex positions indicates that in the diffusion phase, the descent speed is well met by the LES. The vertical positions during the vortex ring phase partially exceed the maximum positions found in the LES (dotted lines). However, the range of vertical extensions of the vortex rings is similar in measurement and prediction. The measured vortex separations (top right) correspond well to the maximum lateral extensions found in the LES during the diffusion phase. Once vortex rings have formed the measured vortex separation depends on the random position of the Lidar measurement plane with respect to the vortex ring. In the presented case linking has probably taken place at $t^{*}=4$, consequently at $t^{*}>4$ the measured horizontal vortex distance can adopt any value as long as it does not exceed the simulated maximum vortex spacing. As shown before circulation varies perceptibly along the vortex line in the ring diffusion phase, so a slight variation of experimental data points is expected and therefore acceptable.

But how can these plots indicate that actually a vortex ring has formed?

1. There is a detection gap of more than two $t_{0}$. Afterwards the vortices are detected again possessing still a significant amount of circulation. The measurements indicate that a headwind of $u^{*}=-1.5\left(\right.$ at $z / b_{0}=3$ ) transported the vortex ring through the Lidar measurement plane. The total axial transport during $2 t_{0}$ amounts to $3 b_{0}$, which is well within the simulated gap length of approximately $6 b_{0}$ at $t^{*}=7$ (see case $\left[N^{*}=0, \epsilon^{*}=0.23\right.$ ] in Fig. 8). These considerations indicate that linking might have occured exactly at the Lidar measurement plane, which then gives significantly lower circulation values as denoted by the single circle symbol at $t^{*}=4.2$.

2. After linking, the lateral distance between both vortices has increased strongly. Figure 8 indicates that elliptic vortex rings perform a quasi-oscillation during which the ring width can exceed the initial length of the vortex ring. The maximal lateral extension of the LES covers the measured lateral vortex separation well, although also shear could play a role regarding vortex ring broadening. Besides very small lateral vortex separations can be observed depending on the random relative position of vortex ring and Lidar measurement plane, see $t^{*}=8.3$ in Fig. 15 .

3. The large scatter of circulation data in the vortex regime can be attributed to two facts. First scatter occurs when the lidar measures vortex segments with identical circulation but different attitudes. 
This automatically leads to an underestimation of circulation. Second, scatter originates from locally varying core radii as demonstrated in Fig. 7 . If it is possible to exclude the measurement uncertainty large data scatter would be a very strong argument for varying core radii which occur during and after linking.

It is concluded that although it cannot be proofed that a vortex ring has formed in the presented case, there are some strong arguements for a successful ring formation which can be noted in several other Awiator cases as well. It will be a challenging task in the future to investigate the frequency of ring formation in detail.

\section{CONCLUSIONS}

LES of aircraft wake vortex evolution in different meteorological conditions have been performed. The impact of the environmental parameters turbulent eddy dissipation rate, turbulence integral length scale, and stable temperature stratification on vortex decay, descent, and topology have been analysed. A special focus was put on the quantitative estimation of vortex deformation, which is believed to be an important factor for the probability and severity of wake vortex encounters.

A new post-processing method has been suggested, which allows identifying significantly eroded vortex core lines and then estimates circulation perpendicular to the current vortex line. In contrast to former results indicating two-phased decay of circulation, the new method reveals that under neutrally stratified conditions circulation decay progresses in three phases. The well-established diffusion phase and phase of rapid decay are followed by a third phase of diffusion, which corresponds to the vortex ring regime. The vortex rings may have substantial lifetimes and descend at a reduced rate compared to the initial parallel vortex pair.

Further it is found that vortex decay in the diffusion phase may depend on turbulence integral length scales provided that these length scales are smaller than the intial vortex spacing. Here, the circulation of the primary vortices is reduced by the work conducted during stretching of environmental eddies. Mutual velocity induction explains the observed vortex topology evolution starting from the initial sinusoidal oscillations to the formation and the subsequent oscillation pattern of the vortex rings. The dimensions of the rings in spanwise direction increase at the expense of the extensions in flight direction. At late stages of wake vortex evolution the majority of the remaining vorticity and circulation is oriented in spanwise direction. Highly variable values for $\Gamma_{5-15}^{*}$ along the vortex ring go along with inversely varying vortex core radii. Qualitative and quantitative good agreement with field measurement data is demonstrated for vortex decay, transport, and topology.

For the first time, vortex deformation is quantified in terms of curvature radii which are inscribed in segments of the vortex line. The established probability distributions of curvature radii correlate with circulation decay. This allows for approximate predictions of curvature radii distributions, which depend on the respective wake vortex circulation and are independent from the prevailing atmospheric conditions. After the rapid decay phase curvature radii of $r_{\kappa} / b_{0}=1$ were shown to dominate the radii distribution.

Considering the significant circulation values and the stability of the ring diffusion phase, the formation of vortex rings should be taken into account in wake vortex prediction systems. Probabilities of the ring formation should be derived, especially in the context that one vortex linking may impede the linking at neighbouring positions due to the increase of horizontal vortex spacing. Knowing typical maximum lengths of straight vortex segments allows to calculate the response of encountering aircraft if it enters the vortices directly. In particular, it should be clarified whether or not vortex rings may pose a hazard for encountering aircraft. Then the safety zones of wake vortex prediction systems introduced by Schwarz and Hahn [40] can be further refined.

\section{FUNDING}

We gratefully acknowledge the financial support of the DLR project Wetter \& Fliegen.

(C) Institute of Atmospheric Physics, DLR Oberpfaffenhofen, Germany 2011

\section{REFERENCES}

1 Delisi, D. P. Laboratory measurements of the effect of ambient turbulence on trailing vortex evolution. In 44th AIAA Aerospace Sciences Meeting and Exhibits, AIAA, San Reno, USA 2006.

2 Holzäpfel, F. Probabilistic two-phase aircraft wakevortex model: further development and assessment. J. Aircraft, 2006, 43(N3), 700-708.

3 Holzäpfel, F., Frech, M., Gerz, T., Tafferner, A., Hahn, K.-U., Schwarz, C., Joos, D., Korn, B., Lenz, H., Luckner, R., and Höhne, G. Aircraft wake vortex scenarios simulation package-WakeScene. Aerosp. Sci. Technol., 2009, 13, 1-11. 
4 Gerz, T., Holzäpfel, F., and Darracq, D. Commercial aircraft wake vortices. Prog. Aerosp. Sci., 2002, 38(N3), 181-208.

5 Crow, S. C. Stability theory for a pair of trailing vortices. AIAA J., 1970, 8(N12), 2172-2179.

6 Donaldson, C. and Bilanin, A. Vortex wakes of conventional aircraft. AGARDograph, 1975, 204.

7 Spalart, P. R. Airplane trailing vortices. Ann. Rev. Fluid Mech., 1998, 30, 107-138.

8 Greene, G. C. An approximate model of vortex decay in the atmosphere. J. Aircraft, 1986, 23, 566-573.

9 Sarpkaya, T. New model for vortex decay in the atmosphere. J. Aircraft, 2000, 37(N1), 53-61.

10 Winckelmans, G., Duquesne, T., Treve, V., Desenfans, O., and Bricteux, L. Summary description of the models used in the Vortex Forecast System (VFS). Univ. Catholique de Louvain, Louvain la Neuve, Belgium, 2005.

11 Holzäpfel, F. Probabilistic two-phase wake vortex decay and transport model. J. Aircraft, 2003, 40(N2), 323-331.

12 Proctor, F. and Hamilton, D. W. evaluation of fasttime wake vortex predictions models. In Proceedings of the 47th AIAA Aerospace Sciences Meeting, AIAA, Orlando, USA, 2009.

13 Melander, M. V. and Hussain, F. Cut-and-connect of two antiparallel vortex tubes. In Proceedings of the Summer Program, Center for Turbulence Research, 1988, N89 24559, pp. 257-286.

14 Kida, S. and Takaoka, M. Vortex reconnection. Ann. Reve Fluid Mech., 1994, 26, 169-189.

15 Kaltenbach, H.-J., Gerz, T., and Schumann, U. Large-eddy simulation of homogeneous turbulence and diffusion in stably stratified shear flow. J. Fluid Mech., 1994, 280, 1-40.

16 Gerz, T. and Holzäpfel, F. Wing-tip vortices, turbulence, and the distribution of emissions. AIAA J., 1999, 37(N10), 1270-1276.

17 Holzäpfel, F. Adjustment of subgrid-scale parametrizations to strong streamline curvature. AIAA J., 2004, 42, 1369-1377.

18 Holzäpfel, F., Gerz, T., and Baumann, R. The turbulent decay of trailing vortex pairs in stably stratified environments. Aerosp. Sci. Technol., 2001, 5(N2), 95-108.

19 Hinton, D. A. and Tatnall, C. R. A candidate wake vortex strength definition for application to the NASA aircraft vortex spacing system (AVOSS), NASA-TM-110343, 1997.

20 Crow, S. C. and Bate, E. R. Lifespan of trailing vortices in a turbulent atmosphere. J. Aircraft, 1976, 13(N7), 476-482.

21 Jeong, J. and Hussain, F. On the identification of a vortex. J. Fluid Mech., 1995, 285, 69-94.

22 Chong, M. S., Perry, A. E., and Cantwell, B. J. A general classification of three-dimensional flow fields. Phys. Fluids, 1990, 5, 050765.

23 Kida, S. and Miura, H. Identification and analysis of vortical structures. Eur. J. Mech. - B/Fluids, 1998, 17, 471-488.
24 Banks, D. C. and Singer, B. A. Vortex tubes in turbulent flows: identification, representation, reconstruction. In Proceedings IEEE Conference Visualization '94, 17-21 October 1994, CP14, pp. 132-139.

25 Hennemann, I. and Holzäpfel, F. Aircraft wake vortex deformation in a turbulent atmosphere. In Proceedings of the 5th International Symposium on Turbulence and shear flow phenomena, TU Munich, Garching, Germany, 2007, pp. 597-602.

26 Hennemann, I. and Holzäpfel, F. Aircraft wake vortex curvature and resulting risk potential for following aircraft. In Proceedings of the CEAS European Air and Space Conference, Manchester, UK, 2009.

27 Proctor, F. and Switzer, G. Numerical simulation of aircraft trailing vortices. In Proceedings of the 9th Conference on Aviation, range and aerospace meteorology, American Meteorology Society, Orlando, USA, 2000, pp. 511-516.

28 Laporte, F. and Leweke, T. Elliptic instability of counter-rotating vortices: experiment and direct numerical simulation. AIAA J., 2002, 40(N12), 2483-2494.

29 Liu, H.-T. Effects of ambient turbulence on the decay of a trailing vortex wake. J. Aircraft, 1992, 29(N2), 255-263.

30 Holzäpfel, F. and Steen, M. Aircraft wake-vortex evolution in ground proximity: analysis and parameterization. AIAA J., 2007, 45(N1), 218-227.

31 Burnham, D. C. and Hallock J. N. Measurements of wake vortices interacting with the ground. In AIAA Paper N98-0593, 1998.

32 Holzäpfel, F., Hofbauer, T., Darracq, D., Moet, H., Garnier, F., and Ferreira Gago, C. Analysis of wake vortex decay mechanisms in the atmosphere. Aerosp. Sci. Technol., 2003, 7, 263-275.

33 Moet, H., Laporte, F., Chevalier, G., and Poinsot, T. Wave propagation in vortices and vortex bursting. Phys. Fluids, 2005, 17, 054109.

34 Holzäpfel, F. Misaka, T. and Hennemann, I. Wakevortex topology, circulation, and turbulent exchange processes. In Proceedings of the AIAA Atmospheric and Space Environments Conference, Toronto, Canada, 2010, AIAA Paper 2010-7992.

35 Saffman, P. G. Vortex dynamics, 1st edition, 1992 (Cambridge University Press, Cambridge).

36 Dhanak, M. R. and Bernadinis, B. The evolution of an elliptic vortex ring. J. Fluid Mech., 1981, 109, 189-216.

37 Sarpkaya, T. Trailing vortices in homogeneous and density-stratified media. J. Fluid Mech., 1983, 136, 85-109.

38 Delisi, D. P. and Robins, R. E. Short-scale instabilities in trailing wake vortices in a stratified fluid. AIAA J., 2000, 38(N10), 1916-1923.

39 de Bruin, A. C. and Kannemans H. Analysis of NLR Cessna citation flight test data for flight test- 1 in the AWIATOR project. AWIATOR, AW-NLR-113-010, NLR, 2004.

40 Schwarz, C. W. and Hahn, K.-U. Full-flight simulator study for wake vortex hazard area investgation. Aerosp. Sci. Technol., 2006, 10, 136-143. 


\section{APPENDIX}

\section{Notation}

$b_{0}$

$c$

$d$

$k$

$L$

$L_{t}$

$L_{t, \boldsymbol{x}}$

$N^{*}$

$p$

$r_{c}$

$r_{\kappa}$

$R$

$t^{*}$ initial vortex spacing $(\mathrm{m})$

cube dimension for corrector calcula-

tion (m)

vector length of predictor step (m)

spectral wave number (-)

computational domain (m)

three-dimensional turbulent integral

length scale $(\mathrm{m})$

one-dimensional turbulent integral

length scales $(\mathrm{m})$

normalized Brunt-Väisälä freqency (-)

pressure $\left(\mathrm{N} / \mathrm{m}^{2}\right)$

vortex core radius $(\mathrm{m})$

curvature radius $(\mathrm{m})$

vortex ring radius $(\mathrm{m})$

normalized time scale $(-)$
$T^{*}$

$u^{*}, v^{*}, w^{*}$

$V_{0}$

$w_{0}$

$\tilde{\mathbf{x}}$

$\tilde{\Gamma}_{5-15}^{*}$

$\Gamma_{5-15}^{*}$

$\Delta$

$\epsilon^{*}$

$\theta$

$\lambda_{\text {Crow }}$

$\sigma$

$\omega$ normalized onset time of linking (-)

wind velocities in axial, horizontal, and vertical direction normalized by $w_{0}(-)$ aircraft flight speed $(\mathrm{m} / \mathrm{s})$

initial vortex descent velocity $(\mathrm{m} / \mathrm{s})$ predicted vortex core line position (m)

normalized circulation along vortex core line (see eqn. 1) (-)

normalized circulation along flight

direction (-)

grid mesh length (m)

normalized turbulent dissipation rate

$(-)$

potential temperature $(\mathrm{K})$

wave length of Crow instability (m)

standard deviation of curvature radii at one instant of time (m)

vorticity $(1 / \mathrm{s})$ 